\title{
Canadian hospitals not doing enough to prevent opioid theft
}

\author{
Cite as: CMAJ 2019 April 23;191:E455-6. doi: 10.1503/cmaj.109-5739
}

Posted on cmajnews.com on Apr. 2, 2019.

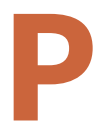

ilferage of opioids by employees is a serious issue at Canadian hospitals, but few have invested resources into preventing the problem. Developers of a new national guideline are hoping to change that.

Data obtained from Health Canada by CMAJ showed that over the last four years, from early 2015 to early 2019, hospital pharmacies reported more than 3000 incidents involving lost or stolen controlled substances, mostly opioids. The majority of the incidents, $84 \%$, were classified as unexplained. Only 213 cases, or $6 \%$, were classified as theft by an employee.

"To me, it's just staggering. People are doing an okay job about finding it, but they're not doing anything to follow up on it," says John Burke, president of the International Health Facility Diversion Association, an international network of health care and law enforcement professionals who share solutions and knowledge on diversion prevention. "Every hospital should have someone who is saying, 'We're not going to tolerate this; we're going to trace down every one of these incidents."'

Burke estimates that hospitals in the United States are also only solving a small fraction of diversion cases. But US hospitals are ahead of Canadian ones when it comes to diversion prevention, according to Sarah Jennings, professional affairs associate at the Canadian Society of Hospital Pharmacists (CSHP).

Hoping to improve hospitals' diversion prevention measures, the CSHP recently released a comprehensive, 28-page guideline with scores of recommendations. Jennings says hospital administration and front-line staff need a "culture

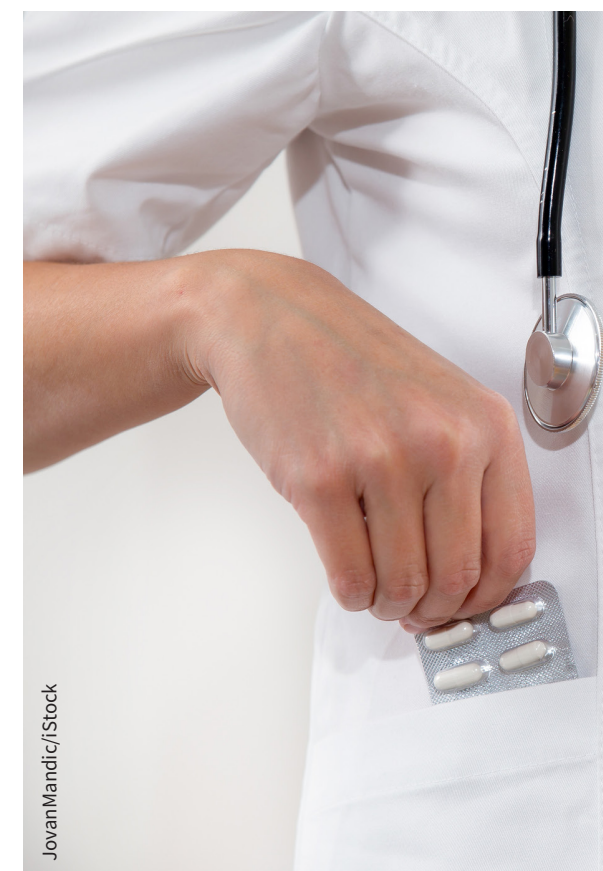

Incidents at hospitals involving missing drugs are more likely to be labelled as unexplained than theft.

shift" to prevent theft. The guideline calls for a dedicated diversion prevention specialist and committee to lead these efforts in each hospital. Diversion prevention committees, which often include representatives from pharmacy, security and nursing teams, are common in major US hospitals, but Jennings isn't aware of a single Canadian hospital with such a committee.

Among numerous other recommendations, the guideline calls for health worker education about the issue, and a twoperson sign-off system whenever a drug is changing hands - from the pharmacy to the patient care area, for example.

Wastage is one of the most common sources for health workers trying to obtain opioids, according to Jennings. For example, nurses are supposed to have another nurse sign off on wastage, but in busy hospitals, nurses may sometimes pocket wastage, planning to find a colleague for sign-off later. "The nurse might end up bringing it home, and think, 'That was easy," Jennings says.

To prevent diversion from wastage, the CSHP recommends that wastage be disposed of before a drug is administered to a patient, and that disposal take place "in an open or observable area."

Another vulnerability, according to Burke, is patients who can have pain medication as needed, Burke says. A hospital employee might say that a patient with dementia needed pain medication 
and end up pocketing it. To prevent this, Burke recommends opioid diversion prevention software, of which there are several options on the market. "With the software, you can run the data to show your top five nurses administering the most pain medication," he says. "It doesn't mean any of them are diverting, but if you see one that's way out of line, that's a red flag to investigate."

Both Jennings and Burke underline that health workers' reluctance to report colleagues is a major barrier. Health workers worry that a colleague's liveli- hood could be taken away. "People might say, 'I don't want them to lose their nursing licence.' Well, do you want them to lose their life, then, because that's a possibility," says Burke. He points out that many health workers who steal opioids are addicted and in need of intervention.

Key parts of any opioid diversion program are addiction prevention and treatment services. In many US hospitals, after an employee caught stealing pharmaceuticals completes a specialized rehab program, which can take two or three years and involve urine screens and security checks, the health worker can have their record wiped clean.

Jennings adds that hospitals need to have a fair, standardized process in place when pilferage is discovered. "You hear of places where one person is given treatment support and allowed to continue working, and another is suspended without pay," she says. "It's important to recognize that this is an illness."

Wendy Glauser, Toronto, Ont. 